\title{
A Modified Streamline-Fractional-Step Finite Element Method for Solving Unsteady Incompressible Viscous Flow*
}

\author{
Eiji MORINAGA** \\ ** Mechanical Engineering, Yawatahama Technical High School \\ 2-3-1, Furu-machi, Yawatahama-shi, Ehime, 796-8003, Japan \\ E-mail: morinaga-eiji@esnet.ed.jp
}

\begin{abstract}
This paper proposes a numerical method for computing the flows at high Reynolds number (Re). A modified fractional step (FS) finite element method (FEM) is based upon the velocity correction method (VCM) and uses two concepts to conduct intermediate velocity. The first is the streamline method, which provides an accurate multidimensional generalization, and the second is the balancing tensor diffusivity (BTD), which is used as the artificial diffusion for the stabilization techniques. The accuracy of this method for the advection-diffusion equation is demonstrated for the rotating cone problem. The unsteady incompressible viscous flows, such as square cavity flow at $\operatorname{Re} \leq 10000$ and flow past a cylinder at $\operatorname{Re} \leq 2000$, are simulated without any numerical instability.
\end{abstract}

Key words : Numerical Method, FS, FEM, VCM, Streamline Method, BTD, Unsteady Incompressible Viscous Flow, High Re

\section{Introduction}

The FEM is an effective method for many engineering problems, such as unsteady, high Re and complicated flows. To numerically stably compute unsteady flow at high Re with high accuracy in the FEM, the upwind method i.e. Petrov-Galerkin method and Taylor-Galerkin metohd, is employed.

A representative of the former is Streamline Upwind Petrov Galerkin method (SUPG) ${ }^{(1)}$ /Pressure Stabilized Petrov Galerkin method (PSPG) ${ }^{(2)}$, which are employed generally to avoid the numerical instability by the advection term of the Navier-Stokes (N-S) equation and the pressure instability by the same-order interpolation for velocity and pressure. However it needs to consider that the added terms in Petrov-Galerkin weighting function will be discontinuous on an element boundary ${ }^{(3)(4)}$, formulations are complicated and stabilized parameters are did various studies ${ }^{(1)(2)}$. Sato et al. ${ }^{(5)}$ propose the Petrov-Galerkin FEM using exponential functions and simulate square cavity flow at $\mathrm{Re} \leq 10^{5}$ by the use of the rectangle element, the mixed interpolation method and the FS method.

The latter is the method in which we use a Taylor-series expansion for time-differential term and introduce second-order-time-differential term as the correction term to make the numerical computation stabilize. This term is called the Balancing Tensor Diffusivity (BTD) term ${ }^{(6)}$. Tanahashi et al..$^{(7)^{\sim}(9)}$ regard the high-order time-integration as the effectiveness in unsteady problems and introduce the BTD term, by using the rectangle element, the mixed interpolation method and the GSMAC method they practice higher-accurate analysis for unsteady flow. Also, about the lumped mass matrix they indicate that the convection term is influenced at initial time of intense flow change, but at later time it is not influenced much ${ }^{(8)(10)}$. Takeuchi and mizuno ${ }^{(4)}$ introduce the BTD term and solve unsteady incompressible viscous flow by using a triangular element, the same-order interpolation of first-order isoparametric element for velocity and pressure and the FS method. To calculate accurately by lumped mass matrix, 
they employ the multi-pass-algorithm (MPA). By using the rectangle element, the mixed interpolation method and the MPA, Kato et al. ${ }^{(11)}$ analyse high-accurate unsteady flow except the effect of numericial viscosity in the upwind FEM by the same method of deriving the BTD term.

We employ geneally the mixed interpolation method ${ }^{(12)}$ to stabilize the calculation in the finite element analysis of imcompressible viscous flow. Takeuchi et al. ${ }^{(4)}$ indicated that this method falls the accuracy of lift and drag calculations but the same-order interpolation method improves these accuracy. Further, the separation solution (for example FS) methods which are solved by separating velocity and pressure fields, are known to stabilize the calculation with the same-order interpolation function. The velocity correction method $(\mathrm{VCM})^{(13)(14)}$ is one of those methods, and it is easy to make the program and use generally.

In this paper, we propose a new modified streamline fractional step finite element method (MS-FS-FEM) to numerically stably simulate unsteady incompressible viscous flows at high Re with high accuracy, and formulate easily in comparison with the SUPG/PSPG method. This method is based upon the VCM and in conducting intermediate velocity we use the streamline method ${ }^{(1)(3)}$ and the BTD term. The streamline method provides an accurate multidimensional generalization and the BTD term is used as the artificial diffusion for the stabilization techniques. To verify the plasticity and validity of the present method, we shall compute the rotating cone problem, square cavity flow and flow past a cylinder.

\section{Basic equations}

For unsteady incompressible viscous flow, the dimensionless basic equations are expressed by the N-S equation and equation of continuity.

$$
\begin{aligned}
& \frac{\partial u_{i}}{\partial t}+u_{j} \frac{\partial u_{i}}{\partial x_{j}}=-\frac{\partial p}{\partial x_{i}}+\frac{1}{R e} \frac{\partial^{2} u_{i}}{\partial x_{j}^{2}} \\
& \frac{\partial u_{i}}{\partial x_{i}}=0
\end{aligned}
$$

where $u_{i}$ is the i-th component of flow velocity, $p$ is pressure, $R e$ is Reynolds number and $t$ is time.

\section{Numerical Analysis}

\subsection{Derivating the BTD term by the Streamline method}

The equation of unsteady advection-diffusion problems by using of scalar-function $\phi$ can be written as:

$$
\frac{\partial \phi}{\partial t}+u_{j} \frac{\partial \phi}{\partial x_{j}}=k \frac{\partial^{2} \phi}{\partial x_{j}^{2}}
$$

where $k$ is a constant diffusion coefficient.

This equation has been expressed as advection-diffusion equation with respect to coordinate $x_{j}$, being carried out the upwind approximation along each co-ordinate $x_{j}$, so the solutions are very poor because of mixing of the crosswind unnecessary artifical diffusion ${ }^{(1)(3)}$. To prevent that, we employ the streamline upwind method which carrys out the upwind approximation along the only flow direction. By dealing the streamline method, we let the co-ordinate $X$ represent the streamline direction and the other co-ordinates represent the normal direction. We derive the BTD term as the advection-diffusion equation with respect to co-ordinate $X$ and the diffusion equation with respect to the other co-ordinates.

Besides, the BTD term is derived by Gresho et al. ${ }^{(6)}$, as will be mentioned below.

$$
\frac{\partial \phi}{\partial t}+U \frac{\partial \phi}{\partial X}=k \frac{\partial^{2} \phi}{\partial X_{j}^{2}}
$$

where $U$ is advective velocity for the streamline direction, being expressed in the form.

$$
U=\sqrt{u_{i}^{2}}
$$


For high Re, forcing $k$ is small and thus Equation(4) may be rewritten as

$$
\frac{\partial \phi}{\partial t} \cong-U \frac{\partial \phi}{\partial X}
$$

Further, assuming that the change of $U$ is small in calculating microscopic time and spaces and $X$ does not depend much on time, we could have

$$
\frac{\partial^{2} \phi}{\partial t^{2}} \simeq U^{2} \frac{\partial^{2} \phi}{\partial X^{2}}
$$

Still more, from computed results, the order of velocity changes are $10^{-2}-10^{-8}$, implying that the prior assumptions are proper.

Next the Taylor-series expansion of $\phi$ with respect to time is given by

$$
\phi^{n+1}=\phi^{n}+\Delta t \frac{\partial \phi^{n}}{\partial t}+\frac{1}{2} \Delta t^{2} \frac{\partial^{2} \phi^{n}}{\partial t^{2}}+O\left(\Delta t^{3}\right) .
$$

By Eq.(4), (7), correcting the second-order time-differential term Eq.(8) can be written as

$$
\frac{\phi^{n+1}-\phi^{n}}{\Delta t} \cong \frac{\partial \phi^{n}}{\partial t}+\frac{1}{2} \Delta t \frac{\partial^{2} \phi^{n}}{\partial t^{2}} \simeq-U \frac{\partial \phi^{n}}{\partial X}+k \frac{\partial^{2} \phi^{n}}{\partial X_{j}^{2}}+\frac{1}{2} \Delta t U^{2} \frac{\partial^{2} \phi^{n}}{\partial X^{2}}
$$

where the third term on the right-hand side is the correction term which is called the BTD term.

\subsection{The $\operatorname{VCM}^{(13)(14)}$ employing the streamline method and the BTD term}

The basic Eq.(1), (2) are discretized as

$$
\begin{aligned}
& \frac{u_{i}^{n+1}-u_{i}^{n}}{\Delta t}+u_{j}^{n} \frac{\partial u_{i}^{n}}{\partial x_{j}}=-\frac{\partial p^{n+1}}{\partial x_{i}}+\frac{1}{R e} \frac{\partial^{2} u_{i}^{n}}{\partial x_{j}^{2}}, \\
& \frac{\partial u_{i}^{n+1}}{\partial x_{i}}=0 .
\end{aligned}
$$

Next we use the intermediate velocity $\tilde{u}_{i}$ as the predictor of velocity, defined in the form

$$
\frac{\tilde{u}_{i}-u_{i}^{n}}{\Delta t}=-u_{j}^{n} \frac{\partial u_{i}^{n}}{\partial x_{j}}+\frac{1}{R e} \frac{\partial^{2} u_{i}^{n}}{\partial x_{j}^{2}} .
$$

This equation is similar with the Euler forward time difference of Eq.(3).

So by Eq.(9), we let co-ordinate $X$ represent the streamline direction, and then employ the streamline method, so that the BTD term Eq.(12) is expressed as

$$
\frac{\tilde{u}_{i}-u_{i}^{n}}{\Delta t} \simeq-U^{n} \frac{\partial u_{i}^{n}}{\partial X}+\frac{1}{\operatorname{Re}} \frac{\partial^{2} u_{i}^{n}}{\partial X_{j}^{2}}+\frac{1}{2} \Delta t U^{n 2} \frac{\partial^{2} u_{i}^{n}}{\partial X^{2}},
$$

or equivalently

$$
\tilde{u}_{i} \simeq u_{i}^{n}-\Delta t\left(U^{n} \frac{\partial u_{i}^{n}}{\partial X}-\frac{1}{2} \Delta t U^{n 2} \frac{\partial^{2} u_{i}^{n}}{\partial X^{2}}-\frac{1}{R e} \frac{\partial^{2} u_{i}^{n}}{\partial X_{j}^{2}}\right) .
$$

The boundary condition (BC) is imposed as

$$
\tilde{u}_{i}=\hat{u}_{i},
$$

where $\hat{u}_{i}$ is known.

Furthermore using Eq.(10), (12), the velocity $u_{i}^{n+1}$ can be written as

$$
u_{i}^{n+1}=\tilde{u}_{i}-\Delta t \frac{\partial p^{n+1}}{\partial x_{i}} .
$$

Taking the divergence on both sides of Eq.(16) and using Eq.(11), we have the pressure Poisson equation

$$
\frac{\partial^{2} p^{n+1}}{\partial x_{i}^{2}}=\frac{1}{\Delta t} \frac{\partial \tilde{u}_{i}}{\partial x_{i}}
$$


This BC for the pressure is

$$
p^{n+1}=\hat{p} .
$$

The algorithm of this method can be summarized as follows.

Step 1 Using the velocity at $n$ time step, by Eq.(14) and BC (15) the intermediate velocity $\tilde{u}_{i}$ is calculated.

Step 2 By Eq.(17) and BC (18) pressure $p^{n+1}$ is calculated.

Step 3 By Eq.(16) velocity $u_{i}^{n+1}$ is calculated.

Step 4 Velocity $u_{i}^{n+1}$ is replaced by $u_{i}^{n}$ and then Steps $1-4$ are repeated.

\section{Finite Element method}

\subsection{Weighted Residual method}

We shall obtain the finite element equations, for Eq.(14),(17),(16). We formulate the space derivation term of these equation by the finite element method. First, using any weighting function $u_{i}^{*}, p^{*}$, these weighted residual equations are given as

$$
\begin{aligned}
& \int_{\Omega} u_{i}^{*} \tilde{u}_{i} d \Omega \simeq \int_{\Omega} u_{i}^{*} u_{i}^{n} d \Omega-\Delta t\left(\int_{\Omega} u_{i}^{*} U^{n} \frac{\partial u_{i}^{n}}{\partial X} d \Omega-\frac{1}{2} \Delta t \int_{\Omega} u_{i}^{*} U^{n 2} \frac{\partial^{2} u_{i}^{n}}{\partial X^{2}} d \Omega\right. \\
& \left.-\frac{1}{R e} \int_{\Omega} u_{i}^{*} \frac{\partial^{2} u_{i}^{n}}{\partial X_{j}^{2}} d \Omega\right), \\
& \int_{\Omega} p^{*} \frac{\partial^{2} p^{n+1}}{\partial x_{i}^{2}} d \Omega=\frac{1}{\Delta t} \int_{\Omega} p^{*} \frac{\partial \tilde{u}_{i}}{\partial x_{i}} d \Omega \\
& \int_{\Omega} u_{i}^{*} u_{i}^{n+1} d \Omega=\int_{\Omega} u_{i}^{*} \tilde{u}_{i} d \Omega-\Delta t \int_{\Omega} u_{i}^{*} \frac{\partial p^{n+1}}{\partial x_{i}} d \Omega .
\end{aligned}
$$

For the third term on the right-hand side of Eq.(19), the second-order differential coefficient term is integrated by parts over the domain $\Omega$, and using the principle of divergence it can be rewritten as

$$
\int_{\Omega} u_{i}^{*} U^{n 2} \frac{\partial^{2} u_{i}^{n}}{\partial X^{2}} d \Omega=\int_{\Gamma} u_{i}^{*} U^{n 2} \frac{\partial u_{i}^{n}}{\partial X} \cdot n_{j} d \Gamma-\int_{\Omega} \frac{\partial\left(u_{i}^{*} U^{n 2}\right)}{\partial X} \frac{\partial u_{i}^{n}}{\partial X} d \Omega
$$

where $\int_{\Gamma}(\quad) d \Gamma$ is zero, because of integration on bounday $\Gamma$ of anaylsis domain $\Omega$. Furthermore, in microscopic spaces, assuming that the change of $U$ is small, Eq.(22) follows that

$$
\int_{\Omega} u_{i}^{*} U^{n 2} \frac{\partial^{2} u_{i}^{n}}{\partial X^{2}} d \Omega=-\int_{\Omega} \frac{\partial\left(u_{i}^{*} U^{n 2}\right)}{\partial X} \frac{\partial u_{i}^{n}}{\partial X} d \Omega \simeq-\int_{\Omega} \frac{\partial u_{i}^{*}}{\partial X} U^{n 2} \frac{\partial u_{i}^{n}}{\partial X} d \Omega .
$$

Similarly second-order differential coefficient term of Eq.(19), (20), those can be obtained as

$$
\begin{gathered}
\int_{\Omega} u_{i}^{*} \tilde{u}_{i} d \Omega \simeq \int_{\Omega} u_{i}^{*} u_{i}^{n} d \Omega-\Delta t\left(\int_{\Omega} u_{i}^{*} U^{n} \frac{\partial u_{i}^{n}}{\partial X} d \Omega+\frac{1}{2} \Delta t \int_{\Omega} \frac{\partial u_{i}^{*}}{\partial X} U^{n 2} \frac{\partial u_{i}^{n}}{\partial X} d \Omega+\frac{1}{R e} \int_{\Omega} \frac{\partial u_{i}^{*}}{\partial X_{j}} \frac{\partial u_{i}}{\partial X_{j}} d \Omega\right) \\
\simeq \int_{\Omega} u_{i}^{*} u_{i}^{n} d \Omega-\Delta t\left\{\int_{\Omega}\left(u_{i}^{*}+\frac{1}{2} \Delta t U^{n} \frac{\partial u_{i}^{*}}{\partial X}\right) U^{n} \frac{\partial u_{i}^{n}}{\partial X} d \Omega+\frac{1}{R e} \int_{\Omega} \frac{\partial u_{i}^{*}}{\partial X_{j}} \frac{\partial u_{i}}{\partial X_{j}} d \Omega\right\},(24) \\
\int_{\Omega} \frac{\partial p^{*}}{\partial x_{i}} \frac{\partial p^{n+1}}{\partial x_{i}} d \Omega=-\frac{1}{\Delta t} \int_{\Omega} p^{*} \frac{\partial \tilde{u}_{i}}{\partial x_{i}} d \Omega .
\end{gathered}
$$

At the end, Eq.(24) can be rewritten by general co-ordinate $x_{i}$ as

$$
\begin{aligned}
\int_{\Omega} u_{i}^{*} \tilde{u}_{i} d \Omega \simeq \int_{\Omega} u_{i}^{*} u_{i}^{n} d \Omega-\Delta t\left\{\int _ { \Omega } \left(u_{i}^{*}\right.\right. & \left.+\frac{1}{2} \Delta t u_{j}^{n} \frac{\partial u_{i}^{*}}{\partial x_{j}}\right) u_{j}^{n} \frac{\partial u_{i}}{\partial x_{j}} d \Omega \\
& \left.+\frac{1}{R e} \int_{\Omega} \frac{\partial u_{i}^{*}}{\partial x_{j}} \frac{\partial u_{i}}{\partial x_{j}} d \Omega\right\} .
\end{aligned}
$$

Here, Eq.(26) is the streamline upwind method employing upwind approximation of advection term for the flow direction. 


\subsection{Interpolation function}

Here dividing two-dimensional analysis domain $\Omega$ into the rectangle elements $\Omega_{e}$, using the shape function $N_{\alpha}$ of four-node rectangle isoparametric element and the same-order interpolations for unknown velocities $\tilde{u}_{i}, u_{i}$ and pressure $p$ can be expressed as

$$
\begin{aligned}
& \tilde{u}_{i}=N_{\alpha} \tilde{u}_{\alpha i}, \\
& u_{i}=N_{\alpha} u_{\alpha i}, \quad(i=1,2, \alpha=1 \sim 4) \\
& p=N_{\alpha} p_{\alpha} .
\end{aligned}
$$

The weighting functions use the same interpolation function of the unknown variables in the Galerkin method.

\subsection{Finite Element equations}

Using the interpolation function on each element $\Omega_{e}$, rewritting the weighted residual equations of Eq.(26), (25), (21), and the finite element equaitions can be derived in the following form

$$
\begin{aligned}
& \tilde{u}_{\beta i} \simeq u_{\beta i}^{n}-\Delta t \bar{M}_{\alpha \beta}^{-1}\left(H_{\alpha j \beta j}+\frac{1}{R e} S_{\alpha i \beta i}\right) u_{\beta i}^{n}, \\
& S_{\alpha i \beta i} p_{\beta}^{n+1}=-\frac{1}{\Delta t} D_{\alpha \beta i} \tilde{u}_{\beta i}, \\
& u_{\beta i}^{n+1}=\tilde{u}_{\beta i}-\Delta t \bar{M}_{\alpha \beta}^{-1} D_{\alpha \beta i} p_{\beta}^{n+1} .
\end{aligned}
$$

Here, the stiffiness matrices can be explained as

$$
\begin{aligned}
M_{\alpha \beta} & =\int_{\Omega_{e}} N_{\alpha} N_{\beta} d \Omega, \\
H_{\alpha j \beta j} & =\int_{\Omega_{e}}\left(N_{\alpha}+\frac{1}{2} \Delta t u_{e j}^{n} \frac{\partial N_{\alpha}}{\partial x_{j}}\right) u_{e j}^{n} \frac{\partial N_{\beta}}{\partial x_{j}} d \Omega, \\
S_{\alpha i \beta i} & =\int_{\Omega_{e}} \frac{\partial N_{\alpha}}{\partial x_{i}} \frac{\partial N_{\beta}}{\partial x_{i}} d \Omega, \\
D_{\alpha \beta i} & =\int_{\Omega_{e}} N_{\alpha} \frac{\partial N_{\beta}}{\partial x_{i}} d \Omega,
\end{aligned}
$$

in which $\bar{M}_{\alpha \beta}$ means the lamped mass matrix obtained from the consistent matrix $M_{\alpha \beta}$ and $u_{e j}^{n}$ is average velocity of element $\Omega_{e}{ }^{(9)}$. Further, the stiffness matrices are required by two-by-two Gaussian quadrature. The pressure Poisson equation of Eq.(29) is solved by the incomplete Cholesky decomposition conjugate gradient method (ICCG).

\section{Numerical examples}

\subsection{Rotating Cone problem}

This problem is the unsteady advection-diffusion problems ${ }^{(1)(11)}$ in two-dimensional rotating flow field shown in Figure 1, and is governed by Eq.(3). To compute the scalar-function $\phi$, whose initial distribution is the cosine function shown in Fig.1, we integrate Eq.(3) in 200 time steps. The accuracy and stability are investigated of the time integral method from the change of that distribution after once rotation of the cone.

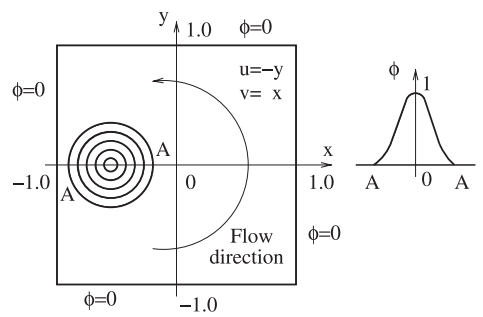

Fig. 1 Rotating cone: Problem statemnet 
Table 1 Cone height

\begin{tabular}{|c|c|c|c|c|c|}
\hline mesh & $\begin{array}{c}\text { Present } \\
\text { method }\end{array}$ & $\begin{array}{c}\text { Artificial diffusion } \\
\text { method }\end{array}$ & $\begin{array}{c}\text { Euler } \\
\text { method }\end{array}$ & SUPG $^{(1)}$ & Kato et al $^{(11)}$ \\
\hline $30 \times 30$ & 98 & 97 & 159 & 88 & - \\
\hline $40 \times 40$ & 99 & 98 & 140 & - & 98 \\
\hline
\end{tabular}

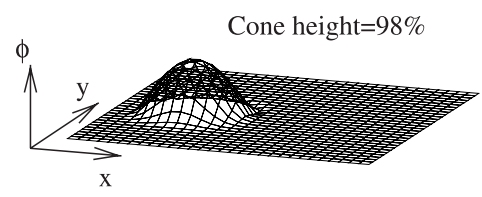

(a) Present method (200 time steps)

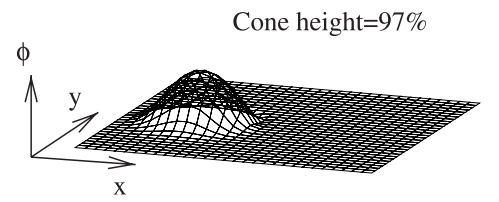

(b) Artificial diffusion method (200 time steps)

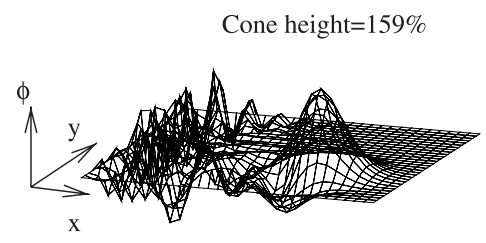

(c) Euler method (80 time steps)

Fig. 2 Rotating cone: Elevations of $\phi$ after once rotation $(30 \times 30$ mesh)

The following methods is compared:

(a) Present method.

(b) Adding artificial diffusion method expressed $\partial \phi / \partial t+u_{j} \partial \phi / \partial x_{j}=\left(k+1 / 2 \Delta t U^{2}\right) \partial^{2} \phi / \partial x_{j}^{2}$.

(c) Euler method.

For high Re, we employ Peclet number $(P e)=10^{6}$ and Courant number $(C r)=0.25$.

Table 1 shows the ratio of cone heights before and after computations. For both mesh sizes, the present method provides better results than other.

The computed cone forms are shown in Fig.2. However, the result of Euler method is shown in 80 time steps because of overflow.

The results of the present method in $30 \times 30$ mesh shows that the cone keeps $98 \%$ of its original height and trailing waves are not abserved. The results of the adding artificial diffusion method shows that the foot of the cone is wider and its height is smaller than the present method. It can be said that the present method accomplishes time-integration with high accuracy.

\subsection{Square Cavity Flow}

The model and BC of square cavity flow are shown in Fig.3. The pressure is set to be zero ${ }^{(5)}$ at the midpoint of the cavity bottom. The rectangle mesh types using the present method are shown in Table 2. Here, the separation of non-uniform mesh is used with method of Okanaga et al ${ }^{(8)}, a$ is non-uniform separate parameter approaching the grid to the wall. The computating conditions of $R e$, microscopic time $\Delta t$, computating time $t$ and mesh type are shown in Table 3.

At $R e=400$, the u-velocity profiles along the vertical center line in square cavity flow is shown in Fig.4. The results of the present method show good agreement with Tezduyar et al. ${ }^{(2)}$. 


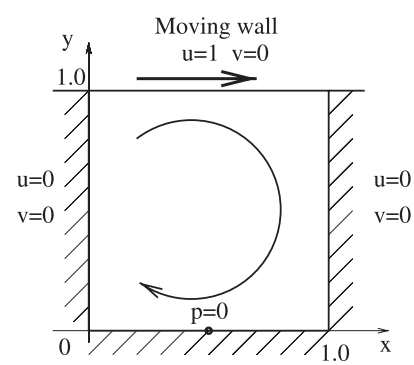

$\mathrm{u}=0 \mathrm{v}=0$
Table 2 Mesh type

\begin{tabular}{|c|c|c|c|c|}
\hline \multirow{2}{*}{$\begin{array}{l}\text { Mesh } \\
\text { type }\end{array}$} & \multirow{2}{*}{ Elements } & \multirow{2}{*}{$a$} & \multicolumn{2}{|c|}{ Mesh size } \\
\hline & & & $\min$ & $\max$ \\
\hline 1 & $32 \times 32$ & - & \multicolumn{2}{|c|}{$\frac{1}{0.0313}$} \\
\hline 2 & $64 \times 64$ & 5 & 0.000574 & 0.0728 \\
\hline
\end{tabular}

Fig. 3 Square cavity model

At $R e=1000-10000$, the velocity profiles along the center line in square cavity flow are shown in Fig.5-9. The velocity profiles show good agreement with Erturk et al. ${ }^{(15)}$. At $R e=10000$, the velocity vectors are shown in Fig.10. As in other investigators ${ }^{(4)(15)}$ results, the secondary vortices appear near the upper-left corner and the lower-left and lower-right corners.

Table 3 Computating conditions

\begin{tabular}{|c|c|c|c|}
\hline$R e$ & $\Delta t$ & $t$ & Mesh type \\
\hline 400 & 0.005 & 50 & 1 \\
\hline 1000 & 0.0005 & 100 & 2 \\
\hline $2500 \sim 10000$ & 0.0005 & 200 & 2 \\
\hline
\end{tabular}

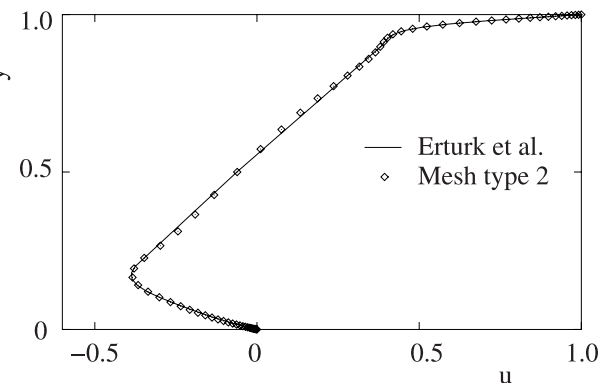

(a) u-velocity

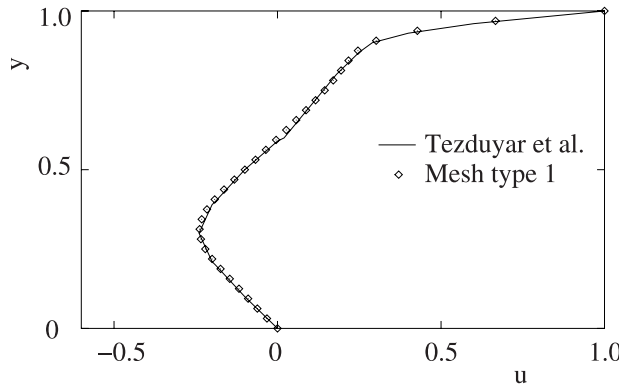

Fig. 4 u-velocity profiles along the vertical center line $(R e=400)$

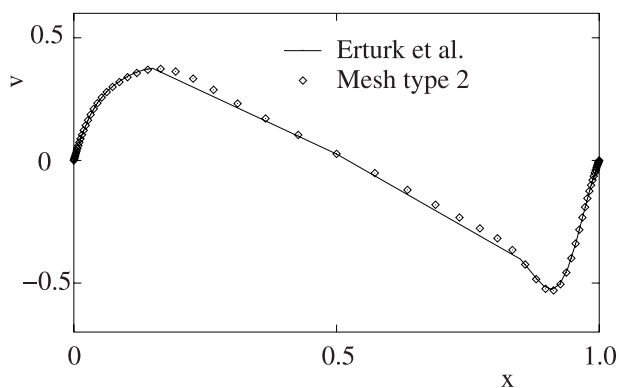

(b) v-velocity

Fig. 5 Velocity profiles along the center line $(R e=1000)$

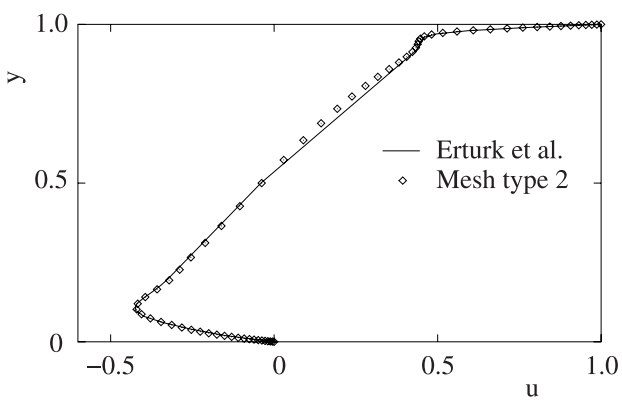

(a) u-velocity

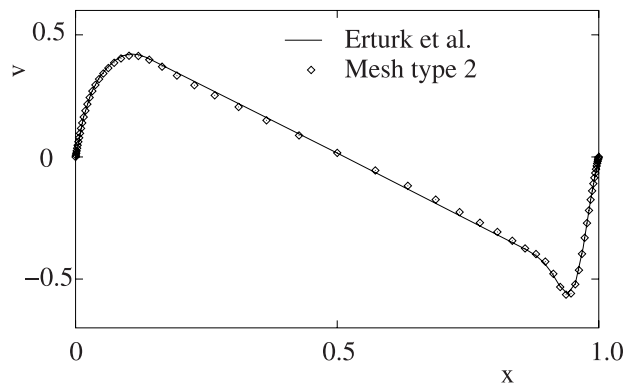

(b) v-velocity

Fig. 6 Velocity profiles along the center line $(R e=2500)$ 


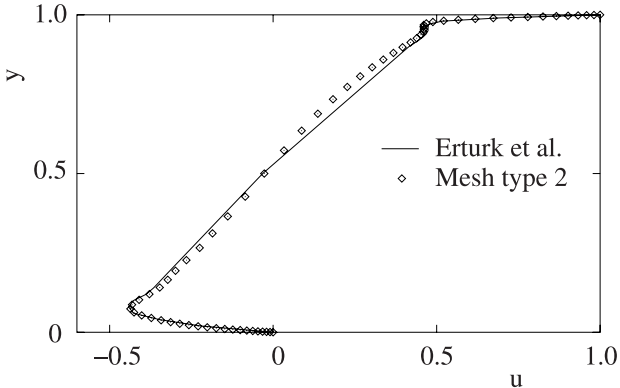

(a) u-velocity

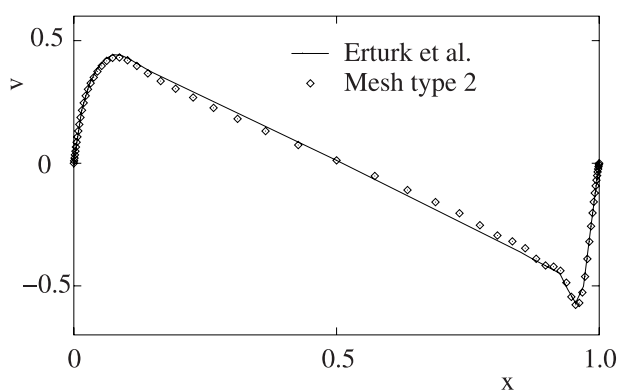

(b) V-velocity

Fig. 7 Velocity profiles along the center line $(R e=5000)$

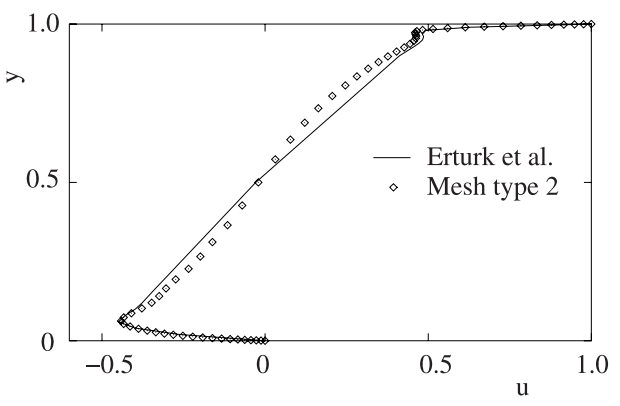

(a) u-velocity

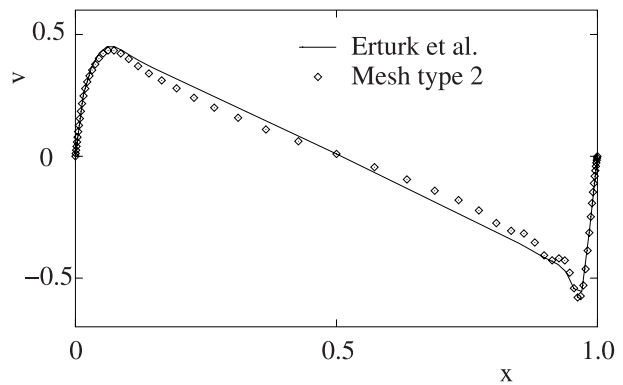

(b) v-velocity

Fig. 8 Velocity profiles along the center line $(R e=7500)$

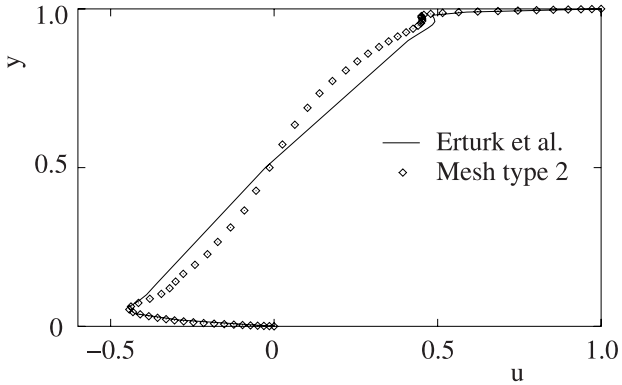

(a) u-velocity

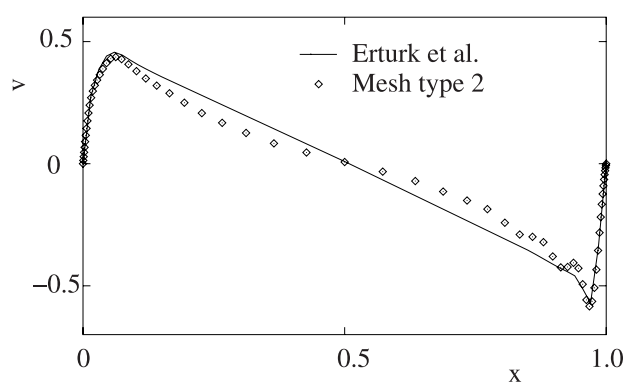

(b) v-velocity

Fig. 9 Velocity profiles along the center line $(R e=10000)$

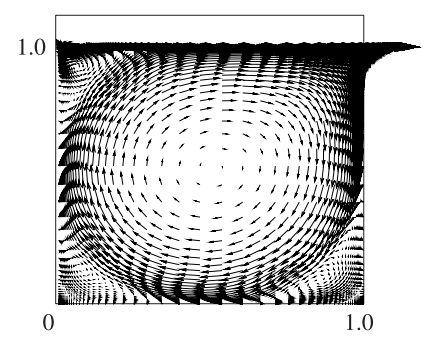

Fig. 10 Velocity vector $(R e=10000)$

\subsection{Flow past a Cylinder}

The separated mesh and BC are shown in Fig.11. Node numbers are 8345 and element numbers are 8200. A minimum value of mesh sizes is 0.0727 and a maximum value is 0.318 . The inlet BC of unit velocity, the outlet BC of zero stress, the up-down BC of $v=0$ and zero 
stress are setted up.

The computating conditions are that $R e$ is 4-2000, $\Delta t$ is 0.001 at $R e=4,10,1400,2000$ and $\Delta t$ is 0.0005 at other $R e$. These simulations are computed untill $t=200$.

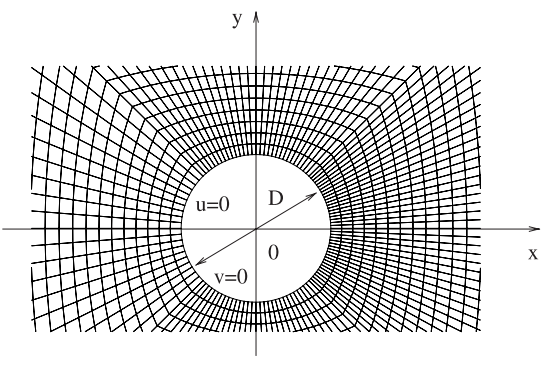

(a) Details of mesh near cylinder

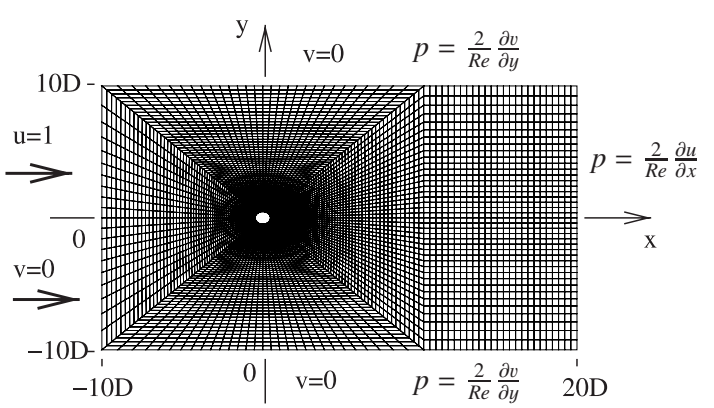

(b) All mesh

Fig. 11 Finite element mesh and boundary condition

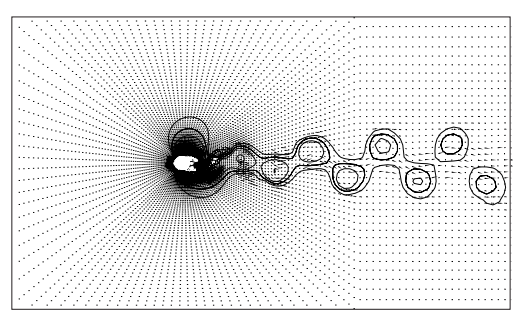

Fig. 12 Velocity vectors and pressure contours $(R e=200)$

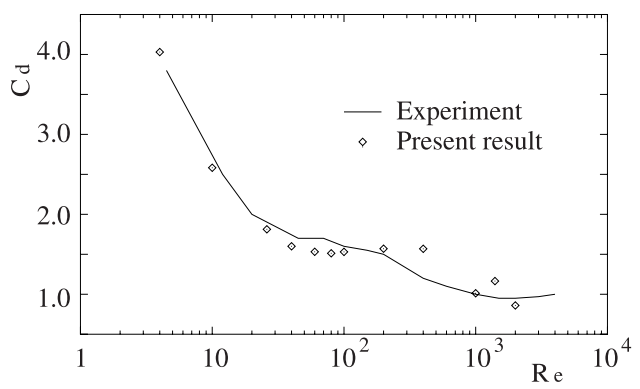

Fig. 14 Relationsip of drag coefficient and Reynolds number

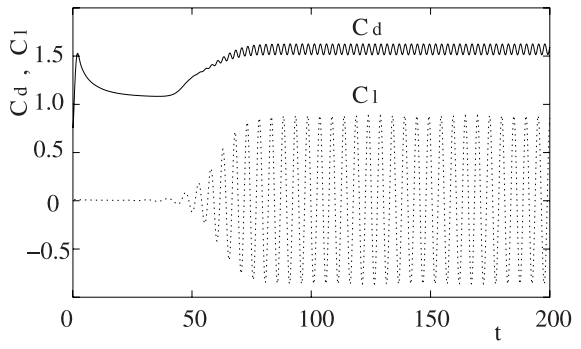

Fig. 13 Time history of the drag and lift coefficient $(R e=200)$

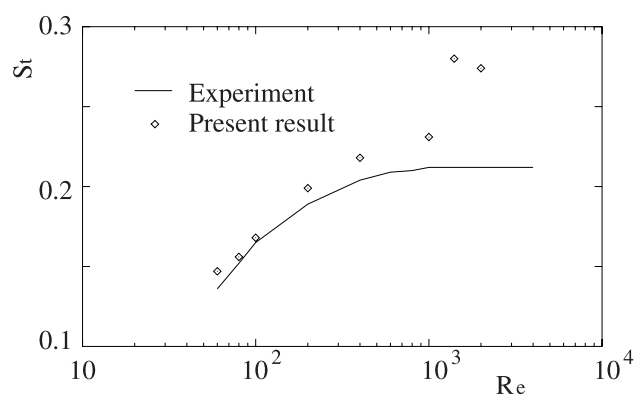

Fig. 15 Relationsip of Strouhal number and Reynolds number

In Fig.12 at $R e=200$ and $t=200$ the velocity vectors and pressure contours, in Fig.13 at $R e=200$ the time history of the drag and lift coefficients $\left(C_{d}\right),\left(C_{l}\right)$ are shown. As in other investigators $^{(1)(2)(10)(11)}$ results, it is shown that the von Kármán vortex street appears periodically.

The relationsip of $C_{d}$ vs. $R e$ and Strouhal number $(S t)$ vs. $R e$ are shown in Fig.14, 15 to compare the present results with the experiment ${ }^{(16)}$. In Re-range of computations, $C_{d}$ decreases with increasing $R e$ and good agreement with experimental results. $S t$ shows agreement with experimental results untill $R e=200$. However at higher $R e, S t$ shows worse agreement because of three-dimensional structure downstream of a cylinder which Kawamoto et al. ${ }^{(10)}$ and Kato et al. ${ }^{(11)}$ reported. 


\section{Conclusions}

In this study, to numerically stably simulate unsteady incompressible viscous flows at high Re with high accuracy, an MS-FS-FEM which can be formulated easily in comparison with the SUPG/PSPG method, is presented and developed for the rotating cone problem, square cavity flow and flow past a cylinder. The following conclusions were obtained:

(1) The time progress method was based upon the VCM, in conducting intermediate velocity employing the streamline method and the BTD term, the flows at high Re were simulated stably.

(2) Adapting the present method to the rotating cone problem, the time-integration was stably done with high accuracy.

(3) Computing square cavity flow untill $R e=10000$, the present results showed good agreement with other investigators results.

(4) Computing flow past a cylinder untill $R e=2000$, the appearance of the von Kármán vortex street was confirmed at $R e \geq 60$, the results of $C_{d}$ and $S t$ showed comparatively quantitative and qualitative agreements with experimental results.

(5) It has been shown that the present method is an effective FEM for stably solving unsteady incompressible viscous flows at high Re with high accuracy.

\section{Acknowledgements}

The author would like to thank professor Genta Kawahara for comments on an earlier draft of the paper.

\section{References}

( 1 ) Brooks, A. N. and Huges, T. J. R., Streamline Upwind/Petrov-Galerkin Formulations for Convection Dominated Flows with Particular Emphasis on the Incompressible Navier-Stokes Equations, Computer Methods in Applied Mechanics and Engineering, Vol.32(1982), pp.199-259.

( 2 ) Tezduyar,T.E. et al., Incompressible flow computations with stbilized bilinear and linear equal-order-interpolation velocity-pressure elements, Computer Methods in Applied Mechanics and Engineering, Vol.95(1992), pp.221-242.

( 3 ) Japan Society of Computaitonal Fluid Dynamics ed., Simulation of the Flow using the Finite Element Method(in Japanese), (1998), p.99, Springer-Verlag Tokyo Publishing CO., LTD.

( 4 ) Takeuchi,N. and Mizuno,A., Finite-Elemnet Analysis of Unsteady Viscous Flow with Same-Order Interpolations, Transactions of the Japan Society of Mechanical Engineers Series B, Vol.61, No.588(1995), pp.118-122.

( 5 ) Sato,H. et al., Petrov-Galerkin Finite-Elemnet Analysis Using Exponential Functions for Square Cavity Flow, Transactions of the Japan Society of Mechanical Engineers Series $B$, Vol.59, No.561(1993), pp.8-15.

(6) Gresho,P.M. et al., A Modified Finite Element Method For Solving The TimeDependent, Incompressible Navier-Stokes Equations. Part 1:Theory, International Journal for Numerical Methods in Fluids, Vol4(1984), pp.557-598.

( 7 ) Okanaga.H. and Tanahashi.T., On High-Order Accuracy of a Numerical Analysis for the Unsteady Advective Equation by Finite-Element Methods, Transactions of the Japan Society of Mechanical Engineers Series B, Vol.56, No.528(1990), pp.228-233.

( 8 ) Okanaga.H. and Tanahashi.T., Numerical Analysis of Natural Convection in a Square Cavity at High Rayleigh Numbers using the GSMAC Finite-Element Methods, Transactions of the Japan Society of Mechanical Engineers Series B, Vol.56, No.530(1990), pp.94-101. 
( 9 ) Jung.C. et al., Development of GSMAC-FEM with Biquadratic Element, Transactions of the Japan Society of Mechanical Engineers Series B, Vol.67, No.653(2001), pp.1-8.

(10) Kawamoto.S. and Tanahashi.T., A High-Speed GSMAC-FEM for Unsteady Incompressible Viscous Flow Analysis, Transactions of the Japan Society of Mechanical Engineers Series B, Vol.57, No.540(1991), pp.168-175.

(11) Kato.C. et al., Time Accurate Solution of Unsteady Viscous Flow Problems using the Finite-Element Method (1st Report, Numerical Scheme), Transactions of the Japan Society of Mechanical Engineers Series B, Vol.58, No.552(1992), pp.300-307.

(12) The Japan Society of Mechanical Engineers ed., Numerical Simulation of the Flow (in Japanese), (1993), p.190, Corona Publishing CO., LTD.

(13) Hayashi, M. et al., Lagrangian Finite Element Method For Free Surface Navier-Stokes Flow using Fractional Step Methods, International Journal Numerical Methods in Fluids, Vol.13(1991), pp.805-840.

(14) Japan Society of Computaitonal Fluid Dynamics ed., Simulation of the Flow using the Finite Element Method(in Japanese), (1998), p.74, Springer-Verlag Tokyo Publishing CO., LTD.

(15) Erturk. E. et al., Numerical Solutions of 2-D Steady Incompressible Driven Cavity Flow at High Reynolds Numbers, International Journal Numerical Methods in Fluids, Vol48(2005), pp.747-774.

(16) The Japan Society of Mechanical Engineers ed., JSME Mechanical Engineers' Handbook A5:Fluid Mechanics, (2004), p.99, Maruzen Publishing CO., LTD. 